\title{
Robust and automated motion correction for real infant fNIRS data
}

\author{
Lindsey J. Powell ${ }^{1,2}$ \\ ${ }^{1}$ Department of Brain and Cognitive Sciences, MIT, Cambridge, MA \\ ${ }^{2}$ Department of Psychology, University of California, San Diego, San Diego, CA
}

\begin{abstract}
Although many approaches have been proposed, removing motion artifacts from developmental fNIRS data remains a difficult challenge. In particular, the lack of consistency in motion correction approaches across experimental reports suggests that the field has not yet identified an algorithm that consistently removes the majority of motion contamination while retaining hemodynamic responses, regardless of the idiosyncrasies of particular datasets. Some existing approaches remove the same fraction of variance from each participant's data; others use participant data to set filtering parameters in ways that result in more stringent thresholds for low-motion participants than high-motion participants. Both types of approach risk leaving artifacts in data from participants with the most motion, while removing signal from participants with the least motion. In contrast, the procedure proposed here identifies and filters motion artifacts on the basis of a fixed, physiologically-justified threshold, so that amount of variance removed is closely associated with the prevalence of motion in each participant's data. Across multiple contrasts from real experimental datasets, this procedure effectively removes motion artifacts while retaining the hemodynamic response signal, allowing the detection of differential responses to conditions, and recovering canonical hemodynamic response functions for both oxygenated and deoxygenated timecourses, indicated by robust negative correlations between the two hemoglobin types. This motion correction procedure would be appropriate to preregister as a planned component of the preprocessing stream in future fNIRS research.
\end{abstract}

\section{Introduction}

Functional near infrared spectroscopy (fNIRS) is a technology that measures the absorption of near infrared light to track changes in blood flow in response to neural activity (Gervain et al., 2011; Meek et al., 1998). It is particularly useful with infant participants, as it is more robust to participant motion than fMRI, the typical method for 
measuring hemodynamic responses in adult brains. However, motion during fNIRS data collection does result in artifacts that need to be removed in order to get a reliable estimate of cortical blood flow.

Many approaches to removing motion artifacts from fNIRS data are motivated by two observations: (1) motion artifacts produce changes in the light intensity signal that are larger and/or more abrupt than those produced by hemodynamic responses to neural function, and (2) motion artifacts account for more shared variance across an array of measurement channels; hemodynamic responses are smaller and more spatially localized. Thus, many motion correction procedures identify and remove signal components characterized by large, rapid changes and/or strong cross-channel correlation (e.g. Wilcox et al., 2005, Molavi \& Dumont, 2012; Yücel, Selb, Cooper \& Boas, 2014; Scholkmann, Spichtig, Muehlemann \& Wolf, 2010). Translating these general strategies into specific implementations that perform well (i.e. remove artifacts while retaining hemodynamic responses) across participants and samples has nonetheless turned out to be a difficult challenge (Brigadoi et al., 2014; Cooper et al., 2012; Goodwin et al., 2016; Behrendt, Firk, Nelson \& Perdue, 2018; Di Lorenzo et al., 2019; Hu et al., 2015; Barker, Aarabi \& Huppert, 2013; Reyes, Bohache, Wijeakumar \& Spencer, 2018).

Much of the difficulty in identifying a specific, reliable approach to motion correction arises from variability across datasets, and within a dataset, across participants, in the amount of motion artifact. Consider motion correction that removes signal components accounting for shared variance across channels (i.e., using principle components analysis (PCA); Wilcox et al., 2005). In a given participant's data, the percentage of variance shared across channels that is truly accounted for by motion will be a function of how much the participant moved, higher for participants who moved a lot and lower for participants who moved little. Remaining shared variance will be a product of 1 ) correlated physiological signals, which researchers likely also want to filter from the timecourse, and 2) common functional properties of the cortical tissue under multiple channels, presumably representing the signal of interest. (The degree to which common functional responses are shared across channels will be affected by the number of channels and spatial extent of the array, which are also dimensions that vary across studies). Thus, picking a fixed percentage of shared variance to remove from all participants' data involves 
a balancing act of removing enough variance to eliminate most motion artifacts in most participants, but not so much that one strips away the hemodynamic responses of interest from low-motion data.

Some approaches attempt to identify and remove motion artifacts specifically, rather than removing any variance shared across channels, by looking for large and rapid signal changes, but these approaches also face challenges in dealing with cross-participant variability in amount of motion artifact. The threshold for changes in signal to be classified as motion is often calculated relative to the variance in each participant's data, e.g. as a multiplier of the global standard deviation or of the interquartile range (IQR) of the wavelet components that make up the signal (e.g. Molavi \& Dumont, 2012; Yücel et al., 2014). This may help motion identification algorithms adjust to differences in baseline signal variability across channels or participants. However, this type of threshold is also impacted by the number and size of motion artifacts present in a given participant's data, often in a counterproductive direction. More frequent and larger motion artifacts increase the standard deviation and IQR. The result is that, for any fixed variance multiplier, the threshold an artifact has to meet to be identified as motion will be higher in a high-motion data set than in a low-motion one. Researchers are once again in the position of needing to pick a threshold low enough that it catches most of the motion for each participant, but not so low that it filters out hemodynamic responses in low-motion data sets.

The fragility of these motion correction approaches in the face of variable data characteristics may explain the wide range of motion correction parameters employed in published infant fNIRS research (Behrendt et al., 2018; Di Lorenzo et al., 2019; Reyes et al., 2018); it has also prompted explicit recommendations to tailor motion correction to specific datasets (e.g. Brigadoi et al., 2014; Reyes et al., 2018). While some kinds of tailoring may be appropriate and easily specified (e.g. using different techniques or parameters to correct data collected from adults vs. infants), a standard practice in which researchers flexibly select a motion correction procedure on a case-by-case basis may increase the likelihood of false positive results, particularly when this selection occurs after data inspection (Gelman \& Loken, 2013; Simmons, Nelson \& Simonsohn, 2011). The efforts described below seek to develop a stable motion correction procedure that reliably reduces artifacts while maintaining hemodynamic responses, and that is robust to participant- and 
experiment-level variation in motion contamination and to differences in array properties typical for infant fNIRS studies. The aim is to provide a procedure that researchers can (1) specify prior to data collection and examination and (2) implement in an automated fashion, without any subjective judgments of data quality or successful motion correction.

We pursued this goal by building a procedure in which motion is identified according to a fixed threshold, defined based on measurement of physiological signals, rather than a parameter estimate that is itself affected by motion prevalence, prior to the application of a targeted PCA filter (Yücel et al., 2014). Specifically, motion artifacts are defined as rapid (i.e. occurring over $1 \mathrm{~s}$ or less) changes in the optical density (OD) form of the data that are 5 or more times greater than the typical amplitude of OD changes produced by the participant's heartbeat. Changes of this amplitude are common for even modest motion artifacts, but exceed the amplitude of most hemodynamic responses, which are typically between 2 and 5 times larger than the heartbeat oscillation (Goodwin et al., 2016), and also typically take more than $1 \mathrm{~s}$ to develop from start to peak (Arichi et al., 2012; Issard \& Gervain, 2018).

This novel procedure was compared with variations of three commonly-used motion correction approaches, encompassing a total of 14 specific motion correction implementations. Each motion correction procedure was applied to two datasets from previously reported infant fNIRS experiments (Powell, Deen \& Saxe, 2018; Powell, Kosakowski, Spelke \& Saxe, 2017), encompassing three condition contrasts of interest. Comparing motion correction procedures on full infant datasets with real, rather than simulated, hemodynamic responses provides three advantages. First, these samples are typical of data from infant participants in that (1) sessions vary substantially in length, sometimes containing the full intended complement of trials but often ending early due to the exhaustion of a participant's attention span, and (2) motion prevalence varied across and within individual participants' data, often increasing substantially toward the end of a session. A motion correction procedure that works well for these data ought to be robust to a degree of between- and within-participant variability, and between-study variability, in motion prevalence that is standard for developmental fNIRS data.

Second, these samples should also exemplify the typical extent to which hemodynamic responses are correlated across multiple fNIRS channels. As reviewed 
above, some motion correction approaches specifically target signal components that explain variance across channels (e.g. PCA filters, Wilcox et al., 2005; Yücel et al., 2014). In tests that involve simulated data, the performance of this type of motion correction algorithm will be particularly impacted by choices regarding the number of channels to which simulated hemodynamic responses are added: the more channels a common simulated response is added to, the more likely the response is to be mistaken for motion and filtered out. Real hemodynamic responses are unlikely to be common to all channels in an fNIRS array (dependent on the extent of channel coverage), and thus adding a simulated signal broadly in a motion correction test may underestimate the performance of sharedvariance approaches (Cooper et al., 2012; Behrendt et al., 2018; Di Lorenzo et al., 2019). On the other hand, adding a simulated signal to only one channel may not realistically reflect the extent to which hemodynamic responses are shared across multiple measurement channels and brain regions, and may thus overestimate the performance of motion correction that removes shared variance. Using real data collected from two different arrays and three experimental contrasts lowers the risk of making incorrect assumptions about the true prevalence of common hemodynamic responses across channels.

Finally, examining the impact of different motion correction procedures on the ability to observe three different experimental effects in two separate datasets increased the likelihood that a procedure that worked well here is generally effective for infants fNIRS data processing, rather than overfit to a particular dataset. For these reasons, motion correction approaches that perform well across the current tests should also reliably achieve the desired outcomes-of removing motion artifacts while retaining hemodynamic response signal-when applied to new infant fNIRS datasets.

\section{Comparison Metrics}

The metrics for assessing successful motion correction were different than those used in previous comparisons of motion algorithms (e.g. Brigadoi et al., 2014; Behrendt et al., 2018; Di Lorenzo et al., 2019; Reyes et al., 2018). Comparisons that proceed by inserting simulated hemodynamic responses into observed or simulated data can test the accuracy with which different procedures recover the known response curve. Here, hemodynamic responses of interest were observed and not simulated, so this evaluation 
approach is not appropriate. Based on previous findings in the literature and initial analyses of the specific datasets in use (Otsuka et al., 2007; Lloyd-Fox et al., 2009;

Grossmann et al., 2008; Naoi et al., 2012; Powell et al., 2018; Powell et al., 2017), each of the tests of interest were expected to yield a positive finding (i.e. an activation difference between conditions), but the exact properties of the true responses were unknown. It was therefore not possible to use error between true and estimated responses as a metric of success. Other comparisons have also assessed which motion correction approaches minimize standard deviation across trials or participants (e.g. Behrendt et al., 2018; Reyes et al., 2018; Di Lorenzo et al., 2019). A concern with this approach is that, while selectively removing motion artifacts would help to reduce signal variability, a reduction in standard deviation could also reflect an unintended flattening of all changes in blood flow that would make it harder to detect true differences in response between conditions, particularly in the context of real but variable hemodynamic responses. Instead, the following three metrics were used to evaluate each approach:

\section{Hemodynamic contrast values}

Effective motion correction of fNIRS data will remove noise introduced by artifacts while retaining the true, underlying hemodynamic responses. When responses to two conditions do reliably differ, the extent to which both of these goals are achieved will be reflected in the $t$ value of a contrast between those conditions, with larger $t$ values suggesting more effective recovery of the true responses. One concern is overfitting: selecting a procedure that maximizes the t value of a single contrast conducted on a single sample could potentially favor an option that leaves behind artifacts and other noise that happen to increase that specific contrast value, or one that removes real but hypothesisinconsistent hemodynamic responses. By searching for a procedure that maximizes $t$ values across multiple contrasts, however, the risk of overfitting is reduced.

Six $t$ values were computed for each motion correction procedure: one oxygenated hemoglobin ( $\mathrm{HbO2}$ ) and one deoxygenated hemoglobin ( $\mathrm{HHb}$ ) contrast value each for comparisons of faces $>$ scenes (Experiment 1, temporal/parietal channels), scenes $>$ faces (Experiment 1, parietal/occipital channels), and imitation > non-imitation (Experiment 2, left medial prefrontal channels). There are three types of relationships between these test statistics: 
(i) Contrast values for the same hemoglobin species but separate experiments (e.g. HbO2 contrasts for faces > scenes and imitation > non-imitation) are fully independent of one another, and thus test the general robustness of each correction procedure.

(ii) $\mathrm{HbO} 2$ and $\mathrm{HHb}$ contrast values for the same comparison (e.g. $\mathrm{HbO} 2$ and $\mathrm{HHb}$ contrasts for faces > scenes) are non-independent, but provide a critical test for the validity of motion correction procedures. During hemodynamic responses to neural activity, the changes in the two species of hemoglobin are typically anti-correlated, while motion artifacts often result in positively correlated changes in both species (Cui, Bray \& Reiss, 2010). Thus a high, positive $t$ value for an $\mathrm{HbO} 2$ contrast that reflects a true difference in responses to the two conditions ought to be accompanied by a substantial, negative $t$ value for the HHb comparison of the same two conditions. If, instead, the $\mathrm{HHb}$ contrast value were also positive, that would suggest both contrast values may reflect remaining motion artifacts that happen to be confounded with condition.

(iii) The face $>$ scene and scene $>$ face comparisons from Experiment 1 were carried out over separate sets of fNIRS channels covering different anatomical regions of interest, but they were also not entirely independent, as the data were collected simultaneously from the same participants and motion correction was applied to all channels concurrently during preprocessing. This relationship had the potential to reveal another way in which motion correction might systematically distort fNIRS data: if a contrast appears reliable because motion filtering was more likely to remove responses to one condition than another (e.g. because responses to one condition were present in a greater number of channels, and thus accounted for more of the shared variance targeted by PCA filters), this would be expected to both weaken the contrast favoring that condition and strengthen the opposing contrast in the remaining set of channels. If a motion correction procedure strengthened both opposing contrasts, however, that would suggest it effectively retained responses to both conditions.

\section{Consistency of functional channel of interest selection}

Activation differences between conditions were identified using an individual functional channel of interest (fCOI) approach (Powell, Deen \& Saxe, 2018). Rather than treating channels in the same array position as equivalent across individuals, subsets of 
each participant's functional data ("selection" data) are used to select the channel, within a predefined anatomical area of interest, most likely to be recording from the functional region of interest (based on its response profile and approximate location). Then responses to the conditions of interest in that channel are compared using the left-out data that did not inform selection. This process is typically repeated over multiple iterations that reassign data to selection and left-out status (e.g. Esterman, Tamber-Rosenau, Chiu \& Yantis, 2010; see Methods for details). This approach can overcome differences in both array-to-anatomy and anatomy-to-function mappings across participants, and substantially improves power to detect group-level differences in both adult and infant samples (Powell et al., 2018).

It is plausible, however, that the individual fCOI approach is quite sensitive to the presence of motion artifacts. Because these artifacts are large relative to hemodynamic responses and infant attention spans dictate that fCOI selection is being carried out over a relatively small number of trials, the presence of artifacts in even just a few trials could disrupt accurate channel selection or reliable estimates of responses from left-out data. In an iterated split-half or leave-one-trial-out procedure, this disruption could be evident in the form of variability in which channel is selected as the fCOI across iterations.

Overcorrecting for motion and removing true hemodynamic responses could also result in variable channel selection, as noise begins to play a greater role than signal in differences between conditions in the selection data. In contrast, cross-iteration channel selection in well-corrected data should be more consistent. (It is not necessary, however, that the same channel be selected across all iterations for the outcome to be valid and interpreted as reflecting the activation from a coherent functional region, not least because multiple adjacent channels could be recording responses from the same underlying region.)

\section{Correlation between oxygenated and deoxygenated hemoglobin}

In response to neural activation, the local volume of $\mathrm{HbO} 2$ increases while that of HHb decreases (Malonek \& Grindvald, 1996; Sheth et al., 2004). As a result, the true timecourses of the two hemoglobin types during a hemodynamic response are strongly negatively correlated (Abdelnour \& Huppert, 2009; Cui et al., 2010). Both undercorrection and overcorrection of motion artifacts may result in this correlation being less negative. Motion artifacts in fNIRS data typically cause both species of hemoglobin to increase or 
decrease in unison, resulting in a positive correlation between the two in undercorrected, motion-contaminated data (Cui et al., 2010). On the other hand, if hemodynamic responses are weakened or removed in low-motion data by an overly stringent motion correction procedure, then the true negative correlation between the two hemoglobin types may shift back toward the zero-correlation state observed in baseline or plateau periods when neither volume is undergoing substantial change. Thus, a well-calibrated motion correction procedure is likely to maximize the negative correlation between $\mathrm{HbO} 2$ and $\mathrm{HHb}$ in activated channels during the stimulus response. Here, we compare the $\mathrm{HbO2}-\mathrm{HHb}$ correlation in the left-out trials from fCOI-selected channels that become each participant's test set for a given contrast.

\section{Comparison Process}

In Phase 1, these three comparison metrics were calculated for data preprocessed and motion-corrected with preexisting functions in the fNIRS analysis package Homer, version 2.2 (Boas, Dubb \& Huppert, 2015). Twelve specific motion correction procedures implemented with these functions (three versions each of a wavelet filter, a PCA filter applied to the whole timecourse, a targeted PCA filter based on a standard deviation threshold, and a targeted PCA filter based on an amplitude threshold) were selected based on their performance in other comparisons of motion correction procedures and their compatibility with the fCOI approach. In Phase 2, additional procedural steps were developed to overcome failure points noticed in the best existing strategy from Phase 1 , implemented via edited versions of Homer2 functions and in-house code, and tested on the same set of comparison metrics.

\section{Phase 1: Comparing existing motion correction procedures}

\section{Methods}

\section{Experiment 1}

One dataset came from a previously reported experiment in which infants watched displays composed of short clips of dynamic face and scene movies (Powell, Deen \& Saxe, 2018, Experiment 2). fNIRS data were collected from right posterior temporal, parietal and occipital cortex while infants viewed these movies. Separate subsets of channels targeted the superior temporal sulcus (STS) and the transverse occipital sulcus (TOS), which are anatomical divisions of cortex known to contain functional regions that respond selectively 
to dynamic faces and scenes, respectively, in adults (e.g. Pitcher et al., 2011; Dilks, Julian, Paunov \& Kanwisher, 2013).

Participants. Participants were recruited from the greater Boston area, and all were full-term (born at 37 weeks of gestation or later). For each participant, a parent or guardian gave written informed consent in accordance with requirements of MIT's Committee on the Use of Humans as Experimental Subjects. Valid data were collected from 16 infants between 3 months, 10 days and 11 months, 5 days of age (mean: 7 months, 3 days; 7 female). One additional infant was excluded for failing to watch a sufficient number of trials.

Materials and procedure. fNIRS data were collected from a 14-channel array using a TechEn CW6 system. The optode at the anterior-inferior corner of the array was positioned at the T4 landmark, with the rest of the array angled along a posterior-superior axis so as to cover large portions of the right temporal, parietal, and occipital cortices (see Figure 1a). Face and scene trials each lasted $15 \mathrm{~s}$, composed of five $3 \mathrm{~s}$ clips, and were interleaved with 6 or $9 \mathrm{~s}$ interstimulus intervals composed of $3 \mathrm{~s}$ clips of scrambled scenes (Figure 1b). These displays were presented in runs, each lasting $85 \mathrm{~s}$, consisting of an animated attention-getter (a spinning star accompanied by a chiming sound) followed by two trials of each condition and three interstimulus intervals. Trial order was counterbalanced across runs (either FSSF or SFFS), which were presented successively until infants became inattentive or fussy. Infants' attention to the displays was later coded from video, and individual trials were rejected if infants failed to attend to the screen for at least two thirds of their duration (10 s). As in the original report of these data, a minimum of six trials per condition were required for inclusion, resulting in the exclusion of one participant. The mean number of valid trials per included participant was 10.4 face trials and 9.1 scene trials. For additional details, see Powell and colleagues (2018, Experiment 2).

Data analysis. Following preprocessing and motion correction (see below), HbO2 and $\mathrm{HHb}$ timecourses were extracted from each valid channel for each trial, beginning $2 \mathrm{~s}$ before the trial onset and extending $4 \mathrm{~s}$ after the offset. Channel-wise responses to each trial were calculated as the mean concentration of each hemoglobin type from $5 \mathrm{~s}$ postonset of the trial to $2 \mathrm{~s}$ post-offset. Tests for preferential responses to faces, relative to 

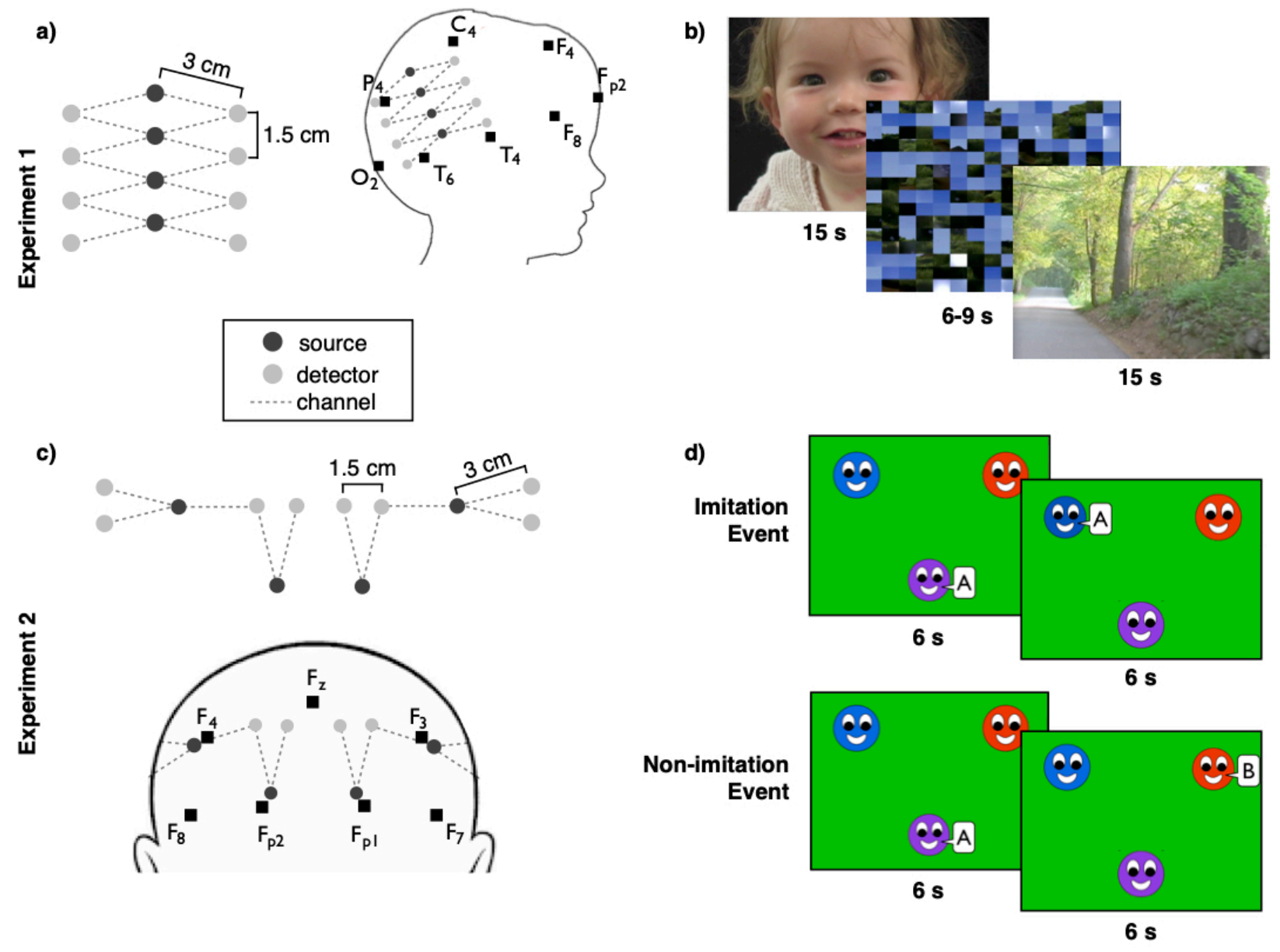

Figure 1. a) Schematic and placement of the fNIRS array inm Experiment 1. b) Sample displays from Experiment 1. c) Schematic and placement of the fNIRS array in Experiment 2. d) Displays from imitation and non-imitation trials in Experiment 2.

scenes, and to scenes, relative to faces, were conducted in separate subsets of channels comprising two anatomical regions of interest (ROIs) using an individual fCOI approach (similar to Powell et al., 2018).

The seven most inferior channels comprised an anatomical ROI targeting right STS, the location of a region of face-preferring cortex in adults (Pitcher et al., 2011). Operating separately on each participant's data from these channels, the fCOI analysis left out responses to a pair of trials (one face, one scene) and used the $\mathrm{HbO} 2$ responses from the remaining trials to select the channel within the STS ROI with the highest $t$ value for the contrast of faces > scenes. The $\mathrm{HbO} 2$ and $\mathrm{HHb}$ responses to the left-out pair of trials from the selected channel were put into a test set. This process was repeated, leaving out a different pair of trials in each iteration. The number of iterations was equal to the number 
of trials in the condition with fewer included trials; if one condition had more valid trials that the other, the remaining, unpaired trials from the more prevalent condition were left out along with the last pair of trials in the final iteration and included in the left-out data contributed to the test set for that iteration. Once all iterations were complete, the participant's mean $\mathrm{HbO} 2$ and $\mathrm{HHb}$ responses for each condition from the left-out test data were analyzed in the group-level data set. Group-level data were used to compute the $t$ values for paired-samples contrasts of faces > scenes for both $\mathrm{HbO} 2$ and $\mathrm{HHb}$ responses. The same procedure was repeated for the contrast of scenes $>$ faces using data from the seven most superior channels, comprising a separate anatomical ROI targeting potential scene preferences in right TOS (Dilks et al., 2013). The maximum proportion of channel selections that could be account for by a single common channel, across all leave-one-pairout iterations, was calculated for each participant and contrast type (faces $>$ scenes and scenes $>$ faces).

The left-out trials from the selected channels (i.e. the trials that made up each participant's group-level contribution) were also used to calculate $\mathrm{HbO} 2-\mathrm{HHb}$ correlations, assessing if these trials showed the expected negative correlation between the two hemoglobin types. Trial-wise correlations were calculated using the $\mathrm{HbO} 2$ and $\mathrm{HHb}$ timecourses from trial onset to trial offset and transformed from $r$ values to a Fisher's $Z$ score. For each contrast, I first calculated participant-level mean $z$ scores across the leftout trial set and then the group-level mean $z$ score, which was converted back to a standard $r$ value for comparison across motion correction procedures.

\section{Experiment 2}

The second data set has not been reported in full, though a subset of participants were presented by Powell and colleagues (2017). Participants in this experiment watched imitative and non-imitative social interactions between animated characters while fNIRS data were collected from lateral and medial prefrontal cortex in both hemispheres. Prior behavioral experiments found that after observing such interactions, infants spend more time looking at and are more likely to reach for the imitator relative to the non-imitating character (Powell \& Spelke, 2017; 2018). The a priori hypothesis was that imitation would elicit higher activation in left medial prefrontal cortex (LMPFC), a region associated with 
social value and approach (e.g. Fox et al., 1995; Grossmann et al., 2008; Naoi et al., 2012; Krueger et al., 2007; van den Bos et al., 2007).

Participants. Participants were 24 full-term infants (age range: 7 mos, 0 days to 9 mos, 30 days; 11 female) from the greater Boston area, whose parent or guardian gave written, informed consent. Ten additional participants were excluded due to unwillingness to wear the fNIRS headgear, inattentiveness to the stimuli, or a lack of sufficient valid fNIRS channels for analysis according to the criteria described below.

Materials and procedure. fNIRS data were collected from an array of channels covering bilateral prefrontal cortex. Two channels each were positioned over four anatomical ROIs: left and right lateral prefrontal cortex (LLPFC and RLPFC), and left and right medial prefrontal cortex (LMPFC and RMPFC; see Fig. 1c). (Data from one channel in each hemisphere connecting the medial and lateral ROIs were not analyzed.) Imitative and non-imitative interaction trials each lasted $12 \mathrm{~s}$. Each interaction began with a center character making a specific sound three times, while jumping up and down. Then one of two side characters would respond by also making three sounds while jumping. One of the side characters was the imitator, and always made the same sound as the center character when responding; the other was the non-imitator and always made a contrasting sound when responding. Infants saw up to four interactions of each type, separated by 6-12 s interstimulus intervals during which geometric shapes moved rigidly on a black background. Looking time to each trial was coded offline, and trials were excluded if infants looked for less than $2 / 3$ of the duration. Infants were excluded if their dataset contained fewer than 3 valid trials per condition.

Data analysis. Following preprocessing and motion correction, $\mathrm{HbO} 2$ and $\mathrm{HHb}$ responses to each trial were calculated as the mean normalized concentration of each hemoglobin species from $8 \mathrm{~s}$ post-onset of the trial to $2 \mathrm{~s}$ post-offset. This time window was chosen to isolate hemodynamic responses to the responding character's imitative or non-imitative action, which began 6 s post-onset and lasted to the end of the trial. An individual fCOI analysis following the same procedure described in Experiment 1 tested for reliably higher activation to imitation vs. non-imitation in the pair of channels positioned over LMPFC. Following the procedures described in Experiment 1, I calculated the maximum frequency at which a single channel was selected across leave-one-pair-out 
iterations, as well as the $\mathrm{HbO} 2-\mathrm{HHb}$ correlations for the left-out imitation trials from the selected channels, from 6s post-onset when the imitation event began, until the end of the trial.

\section{Common preprocessing pipeline}

In addition to motion artifact removal, several additional preprocessing steps are required to transform the raw light intensity data collected by fNIRS systems into the $\mathrm{HbO} 2$ and $\mathrm{HHb}$ timecourses used in estimating hemodynamic responses to neural activity. We held these additional steps constant, so as to isolate the impact of the motion correction procedures. Preprocessing steps were executed using functions from version 2.2 of the HomER NIRS analysis package (Huppert et al., 2009) as follows:

(1) Raw intensity data were transformed into optical density units (hmrIntensity20D). (2) Wavelengths with mean intensity values outside the range of $1 \times 10^{4}-5 \times 10^{6}$ au were pruned (enPruneChannels).

(3) Motion correction was applied to data from valid channels in the OD form.

(4) The corrected data were then bandpass filtered with a high pass filter up to 0.01 $\mathrm{Hz}$ and a low pass filter up to $0.5 \mathrm{~Hz}$ (hmrBandpassFilt).

(5) Data were transformed into $\mathrm{HbO} 2$ and $\mathrm{HHb}$ concentration values using the modified Beer-Lambert law with a partial pathlength factor of 6 (hmrOD2Conc).

\section{Motion correction procedures}

Three motion correction procedures that are commonly used in infant fNIRS studies and/or have performed comparatively well in recent motion correction assessments (e.g. Wilcox et al., 2005; Behrendt et al., 2018; Reyes et al., 2018) were compared to a correction algorithm that is less common, though fully supported by the functions available in version 2.2 of the Homer analysis package. The typical procedures included (1) a wavelet filter (Mohlavi \& Dumont, 2012), (2) a full-timecourse PCA filter (e.g. Wilcox et al., 2005), and (3) a "targeted" PCA (tPCA) filter applied selectively to timepoints identified as motion according to a standard deviation threshold (Yücel et al., 2014). For each of these procedures, I identified the key variable used to adjust the amount of variance identified as motion and/or removed from the timecourse and tested the performance of three levels of this variable, ranging from less to more stringent. For the wavelet filter, the key variable was the multiplier of the interquartile range (iqr) of wavelet coefficients used to identify 
and remove motion wavelets; this value was set at $1.0,0.5$, or 0.1 . For the full PCA filter the key variable was the percentage of shared variance to be accounted for by removed components; this value was set at $90 \%, 95 \%$ or $97 \%$. For the targeted PCA filter, the key variable was the standard deviation multiplier used to set a threshold for identifying periods of motion in each participant's data; this value was set at 25 times, 15 times, or 10 times the standard deviation of the channel's timecourse. The tPCA procedure is typically iterated several times, reidentifying and filtering motion-contaminated timepoints with each iteration; for the tPCA procedure with a varying standard deviation threshold, the number of iterations was fixed a three and components accounting for $97 \%$ of shared variance from motion-affected portions of the timecourse were removed on each pass.

The non-standard procedure was chosen with the goal of better matching the amount of variance removed from a dataset to the amount of motion contamination via a threshold that would not get weaker with increased participant motion, as a standard deviation threshold does. To achieve this, I employed a tPCA filter using an amplitude threshold, rather than a standard deviation threshold. The value for this threshold was based on empirical examination of heart rate signal across the current datasets, which had an amplitude of $\sim 0.02$ OD units in most valid channels (Figure 2a). Given that hemodynamic responses to neural activity are generally 2-5 times larger than the heart

a)

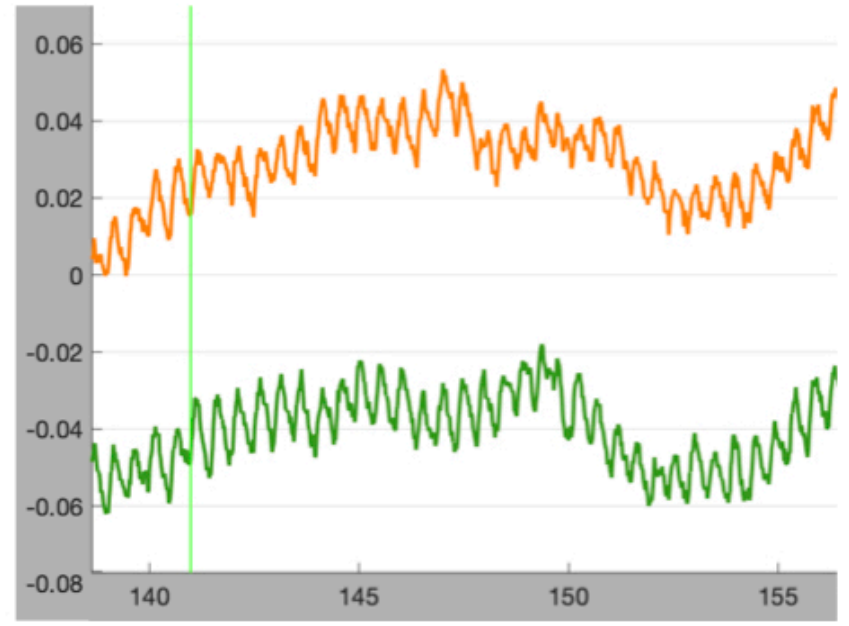

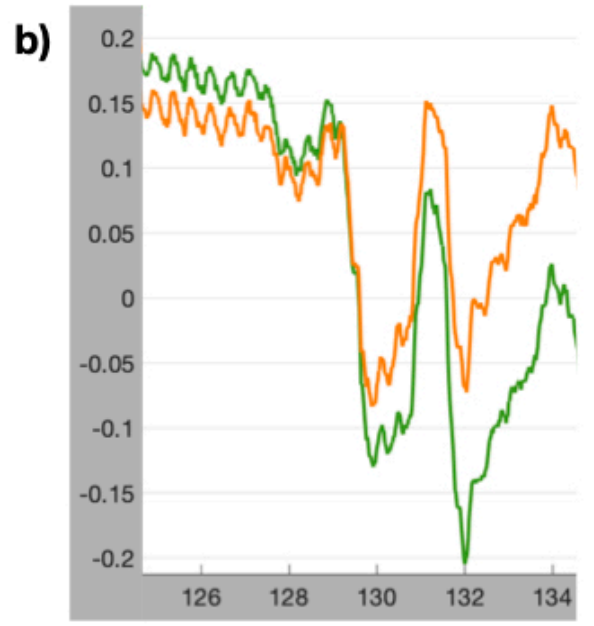

Figure 2. a) Optical density changes for the $690 \mathrm{~nm}$ wavelength from two fNIRS channels, showing steady modulation by heart rate of $\sim .02$ au per beat. b) Rapid and large (>0.1 au) signal changes produced by motion artifacts. 
rate amplitude and that they typically take at least $2 \mathrm{~s}$ from start to peak (Goodwin et al., 2016; Arichi et al., 2012), motion artifacts were defined as any change in signal passing an amplitude threshold of at least 0.1 OD units (i.e. five times the heart rate amplitude) within a $1 \mathrm{~s}$ interval (Figure 2b). To test the optimal stringency of this procedure, the amplitude threshold was held constant but the number of passes of the tPCA filter was varied, employing one, two, or three iterations.

\section{Results}

In an initial set of fCOI analyses, conducted following the preprocessing stream described above but without any form of motion correction, results consistent with the standard hemodynamic response of higher $\mathrm{HbO} 2$ and lower $\mathrm{HHb}$ for each target contrast were weak or absent (Table 1). In Experiment 1, fCOIs in the STS region exhibited significantly higher responses to faces than scenes $(t(15)=2.77, \mathrm{p}=.014)$. However, the $\mathrm{HHb}$ response was also significantly higher for faces than scenes, rather than lower as expected, raising the possibility that both results were the product of condition-correlated artifacts $(t(15)=2.78, \mathrm{p}=.014)$. The scenes $>$ faces contrast in the TOS region showed the predicted directional pattern of higher $\mathrm{HbO} 2$ and lower $\mathrm{HHb}$ for the preferred condition relative to the dispreferred condition, but these differences did not reach the threshold of statistical significance (HbO2: $t(15)=1.67, \mathrm{p}=.12 ; \mathrm{HHb}: t(15)=-1.12, \mathrm{p}=.28)$. The uncorrected analysis for Experiment 2 had a better outcome: the LMPFC fCOIs had a significantly higher $\mathrm{HbO} 2$ response to imitation than to non-imitation $(t(23)=2.14, \mathrm{p}=.04)$ and the $\mathrm{HHb}$ difference was in the expected, negative direction, though it did not pass the standard threshold of statistical significance $(t(23)=-1.94, p=.06)$. Across the three contrasts, within-participant selection of a specific channel as the fCOI was relatively consistent (Table 1), but $\mathrm{HbO} 2$ and $\mathrm{HHb}$ were either weakly negatively correlated or uncorrelated (Table 2). This initial state highlights the need for effective motion correction.

\section{Performance of Motion Correction Procedures by Metric}

1. Hemodynamic Contrast Values. Table 1 reports the $\mathrm{HbO} 2$ and $\mathrm{HHb}$ contrast tvalues for each of the three condition comparisons of interest, following each motion correction procedure. To more easily compare the strength of the t-values generated across contrasts by each motion correction procedure, we also took the mean of the 18 


\begin{tabular}{|c|c|c|c|c|c|c|c|c|c|}
\hline \multirow[t]{2}{*}{$\begin{array}{l}\text { Motion Correction } \\
\text { Method }\end{array}$} & \multicolumn{3}{|c|}{$\begin{array}{c}\text { Faces }>\text { Scenes } \\
\text { (STS) }\end{array}$} & \multicolumn{3}{|c|}{$\begin{array}{c}\text { Scenes > Faces } \\
\text { (TOS) }\end{array}$} & \multicolumn{3}{|c|}{$\begin{array}{l}\text { Imitation > Non- } \\
\text { imitation (LMPFC) }\end{array}$} \\
\hline & $\mathrm{HbO} 2 t$ & $\mathrm{HHb} t$ & $\%$ chan & $\mathrm{HbO} 2 t$ & $\mathrm{HHb} t$ & $\%$ chan & $\mathrm{HbO} 2 t$ & $\mathrm{HHb} t$ & $\%$ chan \\
\hline No correction & 2.77 & 2.78 & $88 \%$ & 1.67 & -1.12 & $80 \%$ & 2.14 & -1.94 & $86 \%$ \\
\hline Wavelet 1.0 IQR & 1.36 & 1.56 & $81 \%$ & 0.69 & -0.59 & $72 \%$ & 1.99 & -1.61 & $85 \%$ \\
\hline Wavelet 0.5 IQR & 1.08 & 1.44 & $81 \%$ & 0.73 & -0.21 & $71 \%$ & 1.88 & -1.53 & $83 \%$ \\
\hline Wavelet 0.1 IQR & 0.71 & 1.67 & $76 \%$ & -0.57 & 0.70 & $63 \%$ & 1.82 & -0.95 & $85 \%$ \\
\hline PCA 90 & 2.14 & -0.13 & $84 \%$ & 2.33 & -1.44 & $83 \%$ & 2.53 & -2.60 & $83 \%$ \\
\hline PCA 95 & 5.00 & -0.32 & $91 \%$ & 2.92 & -1.41 & $83 \%$ & 1.78 & -1.31 & $85 \%$ \\
\hline PCA 97 & 4.88 & -0.62 & $87 \%$ & 4.13 & -1.69 & $86 \%$ & 1.31 & -1.52 & $84 \%$ \\
\hline tPCA-StdThresh 25 & 3.62 & -1.06 & $89 \%$ & 0.39 & -1.05 & $80 \%$ & 2.01 & -2.34 & $85 \%$ \\
\hline tPCA-StdThresh 15 & 1.77 & 0.81 & $83 \%$ & 2.08 & -1.53 & $87 \%$ & 1.11 & 0.21 & $86 \%$ \\
\hline tPCA-StdThresh 10 & 2.80 & -1.54 & $88 \%$ & -0.01 & 0.31 & $86 \%$ & 1.59 & -0.51 & $89 \%$ \\
\hline tPCA-AmpThresh x1 & 3.54 & -0.05 & $90 \%$ & 3.40 & -4.24 & $89 \%$ & 2.73 & -1.16 & $84 \%$ \\
\hline tPCA-AmpThresh x2 & 3.80 & -1.38 & $94 \%$ & 2.90 & -4.05 & $85 \%$ & 2.66 & -1.27 & $89 \%$ \\
\hline tPCA-AmpThresh x3 & 3.01 & -1.34 & $90 \%$ & 2.57 & -3.54 & $85 \%$ & 2.68 & -1.32 & $89 \%$ \\
\hline \multicolumn{10}{|l|}{ Part 2} \\
\hline $\begin{array}{l}\text { tPCA-AmpThresh } x 2 \\
\text { w/ prunePlus }\end{array}$ & 4.15 & -1.64 & $93 \%$ & 2.57 & -3.23 & $86 \%$ & 3.17 & -1.53 & $89 \%$ \\
\hline $\begin{array}{l}\text { tPCA-AmpThresh } \\
\text { Flex w/prunePlus }\end{array}$ & 5.80 & -1.72 & $97 \%$ & 3.04 & -3.98 & $91 \%$ & 3.34 & -1.22 & $84 \%$ \\
\hline
\end{tabular}

Table 1. The six contrast $t$ values following each motion correction procedure, and the percentage of participant-level fCOI channel selections accounted for by a single channel. Bold $t$ values meet the threshold of $\mathrm{p}<.05$ in the hypothesized direction. 
values for each procedure (3 HbO2 values $+3 \mathrm{HHb}$ values, at each of 3 levels of stringency), after reversing the sign for the HHb contrasts so that more negative outcomes raised the average contrast value.

The wavelet method had the lowest mean t-value (0.51), which was in fact lower than the mean t-value for contrasts performed without any motion correction (1.14). Moreover, none of the individual contrasts under the wavelet correction approach reached statistical significance.

The tPCA-StdThresh method ranked third out of the four in overall mean t-value (1.22). The results of individual contrasts were inconsistent, sometimes nearing or meeting statistical significance thresholds but other times falling well short, and no one threshold level $(10 \mathrm{x}, 15 \mathrm{x}$, or $25 \mathrm{x}$ the mean standard deviation) performed well across all three contrasts.

The standard PCA filter ranked second in overall mean t-value at 2.11. At the weakest filter strength (.90), all three $\mathrm{HbO} 2$ contrasts met the threshold for statistical significance at $p<0.05$. At stronger filter settings, however, performance across the three experiments diverged. Stronger filters led to higher contrast values for the face and scene contrasts from Experiment 1, but lower, non-significant values for the imitation contrast from Experiment 2. This was accompanied by a reduction in the amplitude of the $\mathrm{HbO} 2$ response to imitation as the filter strengthened, suggesting that the stronger filter was removing hemodynamic response signal in addition to noise (Figure 3).

The tPCA-AmpThresh method performed best across contrasts, with a mean t-value of 2.53. It did particularly well at revealing reliable $\mathrm{HbO} 2$ differences between conditions: t-values for all contrasts reached the threshold for statistical significance at all levels of stringency (i.e. with 1, 2, and 3 iterations of the filter; see Table 1). HHb differences were less robust, particularly for the face $>$ scene and imitation > non-imitation contrasts. (This was broadly true across motion correction procedures and likely reflects the smaller amplitude of the HHb change in hemodynamic responses; Malonek \& Grindvald, 1996; Arichi et al., 2012). On the basis of contrast values, the tPCA-AmpThresh method was clearly the most robust for removing motion artifacts while retaining elicited hemodynamic responses. One feature of this outcome for researchers to consider is that, 

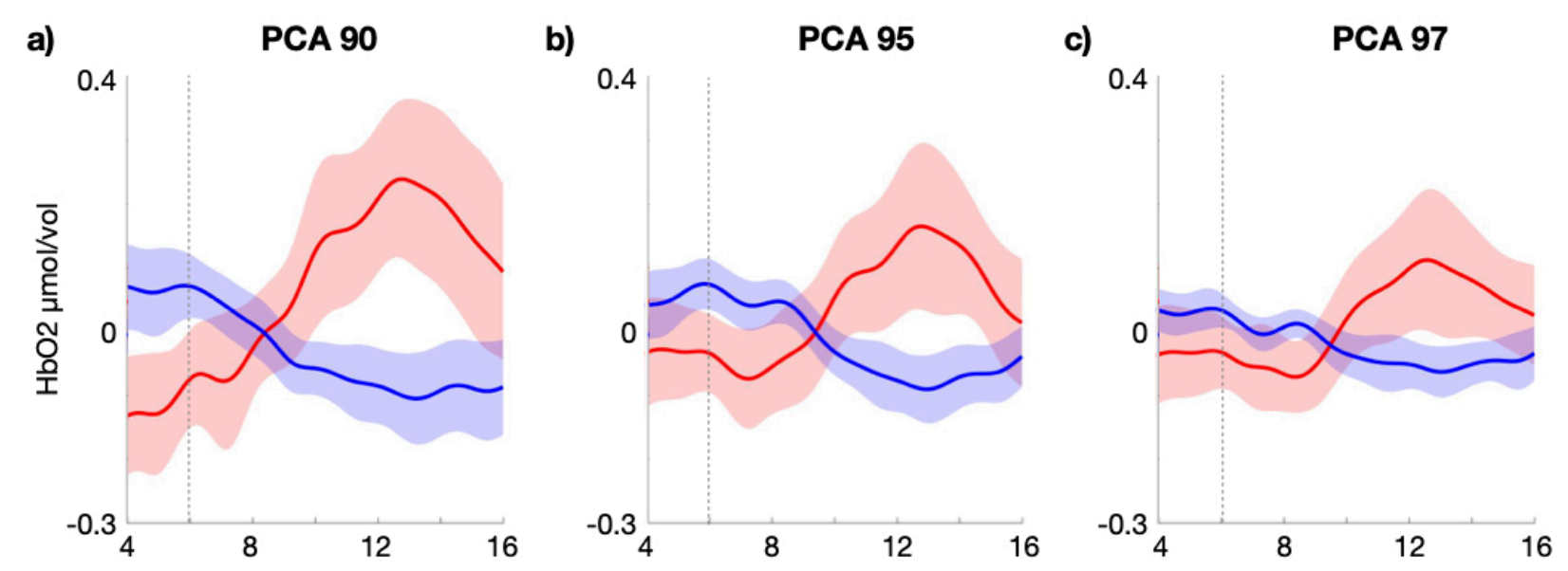

Figure 3. Mean oxygenated (red) and deoxygenated (blue) hemoglobin response to the Experiment 2 imitation events (beginning $6 \mathrm{~s}$ into the trials) in LMPFC, given motion correction with a PCA filter removing $90 \%, 95 \%$, or $97 \%$ of variance shared across trials.

while tPCA filters are typically applied in an iterative fashion, here there was no clear advantage of repeating the filter.

2. Consistency of fCOI selection. On this metric, the wavelet procedure again performed poorly. When compared to no motion correction at all, wavelet filters reduced the consistency with which a single channel was selected as a participant's fCOI for a given contrast across iterations of the leave-one-trial-out procedure (Table 1). Selection consistency did not vary much across the remaining motion correction procedures, though the tPCA-AmpThresh procedure performed best, with the mean percentage of matching channel selections across all contrasts and stringency levels of $88.5 \%$. In combination with the contrast t-values, this outcome shows that the tPCA-AmpThresh procedure allows subsequent fCOI analyses to consistently identify channels that tap functional regions of the infant cortex with reliable response profiles.

Even with no motion correction, channel selection consistency was well above chance ( $84.7 \%$ across the three contrasts with selection from ROIs of 2 to 7 channels), and higher than in the previously reported analysis of Experiment 1 (69\% across face > scene and scene > face contrasts; Powell et al., 2018, SI). Beyond the motion correction procedures, one difference in the present analysis is the use of a leave-one-trial-out approach to the fCOI analysis, rather than a split-half approach. Including more trials in the selection set for each iteration seems to considerably improve selection stability. 


\begin{tabular}{|c|c|c|c|c|c|c|c|c|c|c|c|c|c|c|c|}
\hline \multirow[t]{2}{*}{ Contrast } & \multirow{2}{*}{$\begin{array}{l}\text { No } \\
\text { MC }\end{array}$} & \multicolumn{3}{|c|}{ Wavelet } & \multicolumn{3}{|c|}{ Standard PCA } & \multicolumn{3}{|c|}{ tPCA-StdThresh } & \multicolumn{3}{|c|}{ tPCA-AmpThresh } & \multirow{2}{*}{$\begin{array}{l}\text { Prune } \\
\text { Plus }\end{array}$} & \multirow{2}{*}{$\begin{array}{l}\text { Amp } \\
\text { Flex }\end{array}$} \\
\hline & & 1.0 & 0.5 & 0.1 & 90 & 95 & 97 & $25 x$ & $15 x$ & $10 x$ & $1 x$ & $2 x$ & $3 x$ & & \\
\hline $\begin{array}{l}\text { Faces > } \\
\text { Scenes }\end{array}$ & -.40 & -.57 & -.55 & -.57 & -.78 & -.75 & -.72 & -.63 & -.61 & -.87 & -.72 & -.75 & -.77 & -.77 & -.77 \\
\hline $\begin{array}{l}\text { Scenes > } \\
\text { Faces }\end{array}$ & -.44 & -.49 & -.54 & -.63 & -.66 & -.81 & -.78 & -.71 & -.76 & -.88 & -.74 & -.80 & -.83 & -.67 & -.69 \\
\hline $\begin{array}{l}\text { Imitation > } \\
\text { Non-im. }\end{array}$ & .09 & -.18 & -.42 & -.52 & -.66 & -.74 & -.84 & -.55 & -.88 & -.97 & -.65 & -.67 & -.67 & -.62 & -.64 \\
\hline
\end{tabular}

Table 2. HbO2-HHb correlations in left-out trials from selected functional channels of interest (fCOIs) following each motion correction procedure.

3. Hb02-HHb Correlations. Relative to no motion correction, all motion correction procedures strengthened the negative correlation between $\mathrm{HbO} 2$ and $\mathrm{HHb}$ during responses to the preferred condition in the left-out data from the fCOIs (see Table 2). Wavelet correction was once again the weakest, producing correlations that ranged from $r$ $=-0.178$ to -0.633 . The tPCA-StdThresh procedure again produced highly variable outcomes, ranging from $r=-0.554$ to -0.971 . The standard PCA and tPCA-AmpThresh outcomes largely overlapped, with $r$ values between approximately -0.65 and -0.8 .

One potentially surprising feature of the pattern of $\mathrm{HbO} 2-\mathrm{HHb}$ correlations is that more negative correlations did not always correspond to higher contrast $t$ values. Cui and colleagues (2010) have argued that the HbO2-HHb correlation approaches -1 in uncontaminated fNIRS data and used that value as an underlying assumption in their correlation-based signal improvement (CBSI) approach to motion correction. Here, however, contrast values peaked when the $\mathrm{HbO2}-\mathrm{HHb}$ correlation for preferred trials was between $r=-0.6$ and -0.8 . This range may be a better target when attempting to uncover true hemodynamic responses, as the rate of change during a response may vary more and have a higher maximum for the larger $\mathrm{HbO} 2$ response than the smaller $\mathrm{HHb}$ response 
(Malonek \& Grindvald, 1996; Arichi et al., 2012). Indeed, the HbO2-HHb correlation tended to approach -1 for the strongest iterations of a filter, which also resulted in a reduction of the peak HbO2 response to the preferred condition (Figure 3). This suggests that the strongest negative correlations are produced by overly strong filters that remove a portion of the $\mathrm{HbO}$ signal, making its shape more similar to that of the modestly changing $\mathrm{HHb}$ trajectory.

\section{Discussion}

Many fNIRS motion correction procedures target features of motion artifacts that can, albeit to a lesser extent, also describe the signal changes that accompany hemodynamic responses (e.g. accounting for shared variance in multiple channels or producing changes in hemoglobin concentration that are larger than typical baseline fluctuations). As a result, these procedures have difficulty retaining hemodynamic responses while fully removing artifacts, particularly when the frequency and magnitude of motion artifacts vary considerably across participants, as they do in developmental datasets. This tradeoff was evident in the effect of these procedures on two example datasets: as the stringency of the standard procedures increased, the variability between and across participants' hemodynamic responses decreased, but, in many cases, so did the size of hemodynamic responses and the differences between conditions that the experiments were designed to study.

For PCA filter approaches in particular, the degree of similarity of hemodynamic responses across channels seems to affect how vulnerable those responses are to being removed by the filter along with motion artifacts. In Experiment 1, the array featured more channels (14) and was positioned to capture functional regions with contrasting response profiles to the two conditions presented, one responding to faces but not scenes and the other responding to scenes but not faces. If the data conformed to this prediction, then neither condition would elicit common responses across all channels, and neither type of response would be highly vulnerable to removal by the PCA filter. In contrast, the array used in Experiment 2 featured only eight channels, and, though LMPFC was the only region predicted to display differential responses to imitation and non-imitation, it was plausible that most or all regions would respond during the imitation condition. (Regions of lateral prefrontal cortex respond to statistically structured input and may have encoded the 
similarity between the initiating and imitating characters' movements; Turk-Browne, Scholl, Chun \& Johnson, 2009; Werchan, Collins, Frank \& Amso, 2016). Any widespread response to stimuli from one condition across most or all channels is vulnerable to removal by the PCA filter, which targets signal changes that explain shared variance, and responses to imitation did, in fact, seem to be diminished by standard PCA filtering and tPCA filtering with a standard deviation threshold, particularly as stringency increased (Figure 3). Thus, while a standard PCA filter may often be an appropriate choice to filter motion from infant fNIRS data, researchers should review the properties of their array and the expected responses to each condition across all channels in advance, to consider the degree to which responses are likely to generate shared variance across most or all channels and be vulnerable to removal by the filter. Researchers who use simulated hemodynamic responses to compare performance of motion correction approaches should also consider how choices to add simulated responses to all vs. some NIRS channels may affect the performance of PCA filters in ways that are not necessarily reflective of their performance on real data.

Our comparisons demonstrate that these challenges can be overcome by identifying motion artifacts using a fixed amplitude change threshold. Because motion artifacts produce large, rapid changes in the optical density signal, while hemodynamic responses produce smaller, slower changes, limiting a motion correction filter to the portions of the timecourse with sufficiently large changes minimizes the extent to which the filter removes desired signal. Even when the stringency of the tPCA-AmpThresh procedure was increased by raising the number of filter iterations, the magnitude of the estimated response remained high despite becoming less variable. The amplitude threshold also renders motion a fixed target. When a standard deviation threshold is used to identify motion, the fluctuations that reach that threshold change across tPCA iterations as the mean standard deviation changes, making robust hemodynamic responses more likely to trigger artifact detection in later iterations. In contrast, with an amplitude threshold, new epochs are unlikely to be tagged as motion across subsequent iterations, as the PCA filter will not generally increase the amplitude of a signal change relative to its starting state (though see below for an exception caused by the presence of large spikes). 
Although the tPCA-AmpThresh procedure performed better than more standard motion correction procedures, there were still some possible failure points. Phase 2 aimed to address these by testing several additions to the motion correction procedure on the same metrics used in Phase 1.

\section{Phase 2: Improving and fully automating channel exclusion and motion correction}

Although metrics assessed in Phase 1 indicated that a fixed amplitude threshold can often distinguish motion artifacts from hemodynamic responses, an amplitude threshold can still mistakenly detect motion artifacts when none occurred. Some sources of noise in the fNIRS signal result in the presence of high frequency, high amplitude fluctuations, which, in combination with an amplitude threshold, will lead most or all of a timecourse to be marked as contaminated by motion (Figure 4a). Even if this occurs in only one channel in an array, the tPCA-AmpThresh procedure would then apply a strong PCA filter to the whole timecourse across all channels. As seen in the evaluation of the standard PCA procedure in Phase 1, this can result in a strong reduction in the response signature, especially in small arrays with multiple channels that respond similarly to one or more conditions (e.g. Experiment 2, Imitation condition).

The first change was implemented to prevent the tPCA filter from being erroneously applied to most or all of a timecourse by eliminating these noisy channels. Some noisy channels were already filtered out in the mean intensity-based pruning step included in the previous processing stream, but some were not. To eliminate the remaining cases, we used the amplitude threshold to define affected time periods on a channel-by-channel basis and pruned channels that were defined as $>90 \%$ "motion" contaminated.

The second change also involved an additional criterion for pruning channels: the presence of excessively large spikes in the signal (Figure 4b). If prevalent throughout the timecourse, large spikes would likely trigger standard pruning via an unacceptably high mean intensity level. However, spikes can sometimes inundate a specific channel only part way through an experimental session (e.g. following a motion artifact that affects optode contact with the scalp), or they can occur sporadically throughout a session without elevating the mean intensity to the rejection threshold. The major downside to these massive artifacts is that the principal components that are filtered out in the attempt to remove them can end up creating new artifacts in the reverse direction in neighboring 

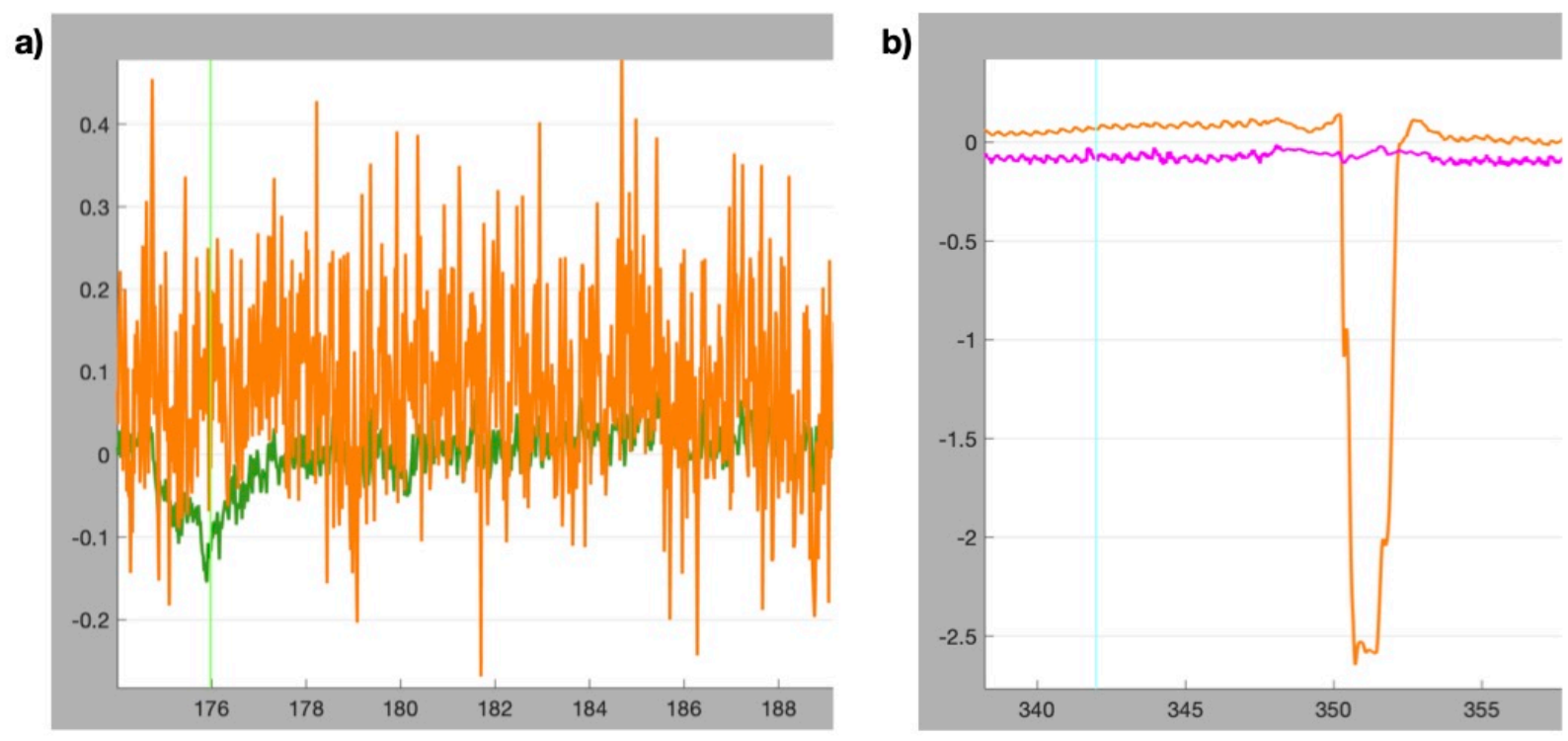

Figure 4. a) High amplitude, high frequency noise that would continually trigger motion artifact detection given the parameters employed by the tPCA-AmpThresh procedure, despite not involving any actual periods of motion. b) An excessively large spike artifact (OD change $>2.0 \mathrm{au}$ ) localized to a single channel.

channels that initially contain similarly-timed but smaller artifacts. The result is that the iterative tPCA filters never satisfactorily remove the artifact, while increasing the disruptive impact of the motion event across channels. To avoid these issues, channels with any spike exceeding 2.0 OD units in amplitude, or 20x the standard motion artifact threshold, occurring during a valid trial, were pruned. (Spikes occurring outside of valid trials were ignored as they could have been the product of large movements affecting many channels, or experimenter adjustment of the headgear, and would also not impede accurate response estimation.)

The third change was prompted by the observation that the ideal number of iterations to use in the tPCA-AmpThresh procedure varied across contrasts and, potentially, across participants. This may reflect the pervasive tradeoff between removing artifacts and removing signal. Even though the use of an amplitude threshold helps to avoid misidentifying hemodynamic responses as motion artifacts (as could occur when using a stringent standard deviation or wavelet threshold on a low motion data set), PCA filters still have the potential to remove signal related to a hemodynamic response that cooccurs with an artifact. The relative prevalence of motion in an individual participant's 
data set, both before motion correction and between tPCA iterations, may help adjudicate when artifact removal is likely to be more helpful or more harmful in discovering a reliable response profile. To test this possibility, the prevalence of motion artifacts in a participant's most contaminated channel was compared to a threshold of $10 \%$ in order to determine whether or not to execute a(nother) tPCA iteration, resulting in a minimum of zero iterations and a maximum of three iterations.

A final manipulation assessed the advantage of excluding contaminated timepoints. Some analyses have investigated the impact of excluding whole trials on the basis of any artifact and have found that, in the context of infant NIRS data, trial exclusion is less helpful than correction and inclusion (e.g. Behrendt et al., 2018; di Lorenzo et al., 2019), probably due to the limited amount of uncontaminated data it is possible to collect with infant participants. Given that motion artifacts can be brief relative to the duration of a trial consisting of blocked stimuli, the current procedure tested the exclusion of affected timepoints, rather than whole trials, on a channel-by-channel basis, based on the same amplitude threshold used to identify motion epochs for the tPCA procedure.

The impacts of these four changes were evaluated on the same metrics used in Phase 1, in semi-cumulative fashion. The first step tested the impact of the two additional channel pruning criteria (prevalence of high amplitude noise, triggering the motion detection algorithm; and presence of large spikes during trials), relative to the basic tPCAAmpThresh procedure utilized in Phase 1. The next step added the capacity to flexibly adjust the tPCA iteration number in response to motion prevalence, as well as the omission of motion-contaminated timepoints, and again calculated performance according to the same metrics used in Phase 1.

\section{Methods}

The data, preprocessing, and analysis were the same as those used in the tPCAAmpThresh procedure used above, except as follows:

1) For the "prunePlus" version of this motion correction approach, the two new criteria outlined above were added to the channel pruning step of the preprocessing pipeline. First, the hmrMotionArtifactByChannel function from Homer2 was used, with the same parameter settings as the tPCA-AmpThresh procedure (tMotion $=1.0$, tMask $=1.0$, Std Thresh $=100$, Amp Thresh $=0.1$ ), to identify channels for which $>$ 
$90 \%$ of timepoints were marked as motion contaminated and pruned these channels from further analysis. Second, hmrMotionArtifactByChannel was used again, this time with an Amp Thresh setting of 2.0, and applied only to timepoints from valid trials, to identify and prune any channels with excessively large motion artifacts occurring during responses of interest.

2) For the "AmpFlex" version of the motion correction approach, a step was added prior to each iteration of the tPCA filter in which hmrMotionArtifactByChannel was used to calculate the proportion of timepoints marked as motion for each channel, using the same parameter settings as the tPCA-AmpThresh procedure (see above). If motion affected fewer than $10 \%$ of timepoints in all channels, then the motion correction procedure was terminated. The maximum number of iterations, regardless of motion levels, was set at three, so each participant's dataset was iteratively filtered between 0 and 3 times, depending on the initial prevalence of artifacts and the effectiveness of each iteration of filtering. Following the tPCA filter, hmrMotionArtifactByChannel was used again to identify timepoints that remained contaminated by motion artifacts and excluded these from channel-wise time courses by replacing the concentration values with $\mathrm{NaN}$.

The same comparison metrics as in Phase 1 were calculated for each of the three contrasts using 1) the new prunePlus procedure followed by tPCA-AmpThresh set to two iterations, and 2) the prunePlus procedure followed by tPCA-AmpFlex. The outcomes for each of these approaches were compared to the mean results of standard pruning and tPCA-AmpThresh in Phase 1.

\section{Results}

Across the two experiments, 8 of the 40 participants had channels that were rejected for having $>90 \%$ of timepoints flagged by the motion detection algorithm set to the standard 0.1 au amplitude threshold. Two participants had channels that were rejected for having artifacts detected during valid trials when the motion detection algorithm was set with a particularly high amplitude threshold of $2.0 \mathrm{au}$.

\section{Performance of Motion Correction Procedures by Metric}

1. Hemodynamic contrast values. Relative to the mean $t$ values (sign-reversed for HHb contrasts) across the tPCA-AmpThresh procedures in Phase 1 (2.53), adding the 
prunePlus procedures for excluding channels resulted in only a slight overall improvement in contrast t-values ( $M=2.71$ across all six contrasts; see Table 1$)$. The largest improvement was evident in the $\mathrm{HbO} 2$ contrast for imitation > non-imitation (maximum $t$ value from Phase 1: 2.73, $\mathrm{p}=.01$; prunePlus $t$-value: $3.17, \mathrm{p}=.004$ ), perhaps because this was the contrast that was most sensitive to high-amplitude, high-frequency noise triggering "targeted" PCA filtering of the full timecourse.

The overall improvement of the contrast values was more evident following the shift to the tPCA-AmpFlex procedure, which yielded a mean $t$ value of 3.18 across the six contrasts. For three of the contrasts (both face > scene contrasts, and the HbO2 contrast for imitation > non-imitation), this procedure yielded the highest $t$ value of all tested motion correction procedures, and performance was also strong relative to other procedures for the remaining three contrasts.

2. Consistency of fCOI selection. Both of the new procedures were similarly strong in channel selection consistency compared to the tPCA-AmpThresh results in Phase 1 (Table 1). The combination of prunePlus and tPCA-AmpFlex provided the highest mean selection consistency of all procedures tested (91.0\%).

3. Hb02-HHb correlation. As in the tPCA-AmpThresh results from Phase 1, the correlation between the two hemoglobin types in selected fCOIs for left-out trials from the preferred condition ranged between -.6 and -.8, rather than approaching -1 (see Table 2).

\section{Discussion}

The additional steps introduced in Phase 2, which focused on 1) eliminating noisecontaminated channels that might interact poorly with tPCA filtering and 2) increasing the sensitivity of the procedure to participant-level motion prevalence, resulted in modest but relevant gains in performance on the contrast value metric. The introduction of these steps never affected whether a contrast passed the significance threshold of $\mathrm{p}<.05$, but they did improve the rate at which contrasts passed the $\mathrm{p}<.005$ threshold some have suggested as a better alternative to protect against Type 1 errors (Benjamin et al., 2018). Following the combined prunePlus and tPCA-AmpFlex procedures, all three $\mathrm{HbO} 2$ contrasts met this stringent criterion (for a directional, one-tailed test in the case of the scene $>$ face contrast). The two HHb contrasts that did not meet the $\mathrm{p}<.05$ threshold following these procedures failed to reach significance following any motion correction. Thus, I conclude that these 
procedures reflect the best practice for detecting group-level differences between conditions in real fNIRS data collected from preverbal infants using variable paradigms and headgear designs.

Performance on the remaining two metrics did not differ much from the results of the tPCA-AmpThresh procedures in Phase 1. Channel selection consistency for those procedures was already nearing $90 \%$ and shifted slightly higher following the introduction of the prunePlus and AmpFlex adjustments. The fact that the HbO2-HHb correlations remained in a similar range and did not become more negative despite the increase in mean contrast $t$-value again suggests that the correlation between the hemoglobin types in optimally recovered hemodynamic responses does not actually approach $r=-1$, as assumed by some motion correction approaches (Cui et al., 2010).

\section{General Discussion}

The goal of this research was to test specific implementations of and alterations to existing fNIRS motion correction procedures in order to identify an approach that could be pre-specified (prior to viewing data) and automatically applied in the context of any fNIRS experiment with preverbal infant participants. It sought algorithms that would maintain a similar level of sensitivity to motion artifacts regardless of their prevalence across participants or experiments, and that would prioritize maintaining the signal from hemodynamic responses in addition to removing motion artifacts, even when responses were widely shared across channels in an array. This search process arrived at a procedure that assesses whether motion correction is necessary and, if so, directs targeted PCA filtering to regions flagged as containing motion according to a fixed, physiologicallyjustified amplitude threshold. This procedure also uses tailored channel pruning to remove channels with poor signal quality and sources of noise likely to interact poorly with the filtering approach. This procedure was tested, along with a range of alternatives, on its ability to recover differences in hemodynamic responses to a range of experimental conditions in a variety of cortical regions of interest. I also tested the consistency with which the participant-level loci of such differences could be detected, and the recovery of a canonical hemodynamic response function, as indicated by a robustly negative $\mathrm{HbO} 2-\mathrm{HHb}$ correlation. 
This novel approach to fNIRS motion correction was successful across all three metrics: it reliably detected differences in $\mathrm{HbO} 2$ responses to experimental conditions in three contrasts, consistently picked out the same functional channels of interest as the loci of those differences, and recovered robust negative correlations between $\mathrm{HbO} 2$ and $\mathrm{HHb}$ timecourses in those fCOIs. Across these metrics, the novel approach performed better than a number of other procedures that are either commonly used in infant research (e.g. standard PCA filters; Wilcox et al., 2005; Emberson et al., 2017; Hyde, Boas, Blair \& Carey, 2010; Powell et al., 2018) or that have been recommended by other recent motion correction evaluations (e.g. wavelet filters; Brigadoi et al., 2014; Behrendt et al, 2018; di Lorenzo et al., 2019). The wavelet filter approach, at all three tested levels of stringency, performed particularly poorly, which is noticeable given its success in comparisons that have used simulated data and/or looked primarily for broad reductions in signal variance following motion correction. The novel tPCA-AmpThresh procedure does bear some resemblance to a recently suggested procedure that combines a slightly more stringent amplitude threshold ( $0.4 \mathrm{au}$ ) and slightly less stringent standard deviation threshold (50x) than typical tPCA filtering, which outperformed other motion correction procedures on a different set of metrics in the context of fNIRS data from young children (Reyes et al., 2018). Future efforts could directly compare these two approaches, as well as comparing the currently supported procedure to more recently suggested methods and approaches, that include different filtering algorithms (e.g. Savitzky-Golay filters; Jahani et al., 2018) or the combination of multiple correction approaches (e.g. Di Lorenzo et al., 2019).

One potential concern is that, by explicitly attempting to maximize statistical contrast values, the current comparisons may have prioritized a motion correction procedure that is overfit to the specific test data. However, the hemodynamic contrast metric tested the ability of correction procedures to maximize $t$-values across six different contrasts, including two contrast pairs that prioritized opposite condition differences (Experiment 1, face $>$ scene contrasts and scene $>$ face contrasts). Thus, a motion correction procedure that inflated responses to one particular condition would not have strengthened one contrast value but left others either weakened or unaffected. The bestperforming procedure produced notably strong contrast values across datasets, and thus does not seem to be overfit. 
A related concern is that the motion correction procedure developed here will be overfit to experimental data with particular characteristics. However, the two experimental datasets used in the current comparison differ in many respects including the average length of the timecourses (Experiment 1: 9 minutes, Experiment 2: $<5$ minutes), the duration of the events of interest (Experiment 1: 15 seconds, Experiment 2: 6 seconds), the regions targeted for measurement (Experiment 1: STS and TOS, Experiment 2:

prefrontal cortex), the ages of participants (Experiment 1: 3-11 months, Experiment 2: 7-9 months), the number of channels (Experiment 1: 14, Experiment 2: 8), and the prevalence of a common signal across most channels. The data used in the current comparisons are also more likely to be representative of infant fNIRS data than in other cases simply by virtue of being real data rather than simulated hemodynamic response functions.

In sum, I suggest that the motion correction procedure developed and tested above is likely able to successfully extract hemodynamic response signal, while discarding motion artifacts, from a broad range of infant fNIRS datasets. It should thus be an appropriate motion correction procedure to preregister and/or apply without any dataset-specific tailoring or adjustments. Future research should test this proposal, and also assess the performance of a similar approach with samples of different ages and data collection conditions.

\section{Acknowledgments}

The author thanks Hilary Richardson and Rebecca Saxe for feedback on an earlier draft of this manuscript. This work was supported by NSF award 1627068.

\section{References}

Abdelnour, A. F., \& Huppert, T. (2009). Real-time imaging of human brain function by nearinfrared spectroscopy using an adaptive general linear model. NeuroImage, 46(1), 133-143.

Arichi, T., Fagiolo, G., Varela, M., Melendez-Calderon, A., Allievi, A., Merchant, N., ... Edwards, A.D. (2012). Development of BOLD signal hemodynamic responses in the human brain. NeuroImage, 63, 663-673. 
Barker, J. W., Aarabi, A., \& Huppert, T. J. (2013). Autoregressive model based algorithm for correcting motion and serially correlated errors in fNIRS. Biomedical Optics Express, 4(8), 1366-1379.

Behrendt, H. F., Firk, C., Nelson III, C. A., \& Perdue, K. L. (2018). Motion correction for infant functional near-infrared spectroscopy with an application to live interaction data. Neurophotonics, 5(1), 015004.

Benjamin, D. J., Berger, J. O., Johannesson, M., Nosek, B. A., Wagenmakers, E. J., Berk, R., ... \& Cesarini, D. (2018). Redefine statistical significance. Nature Human Behaviour, 2(1), 6-10.

Brigadoi, S., Ceccherini, L., Cutini, S., Scarpa, F., Scatturin, P., Selb, J., ... Cooper, R.J. (2014). Motion artifacts in functional near-infrared spectroscopy: A comparison of motion correction techniques applied to real cognitive data. NeuroImage, 85, 181-191.

Cooper, R.J., Selb, J., Gagnon, L., Phillip, D., Schytz, H.W., Iversen, H.K., ... Boas, D.A. (2012). A systematic comparison of motion artifact correc- tion techniques for functional near-infrared spectroscopy. Frontiers in Neuroscience, 6, 147.

Cui, X., Bray, S., \& Reiss, A. L. (2010). Functional near infrared spectroscopy (NIRS) signal improvement based on negative correlation between oxygenated and deoxygenated hemoglobin dynamics. NeuroImage, 49(4), 3039-3046.

Di Lorenzo, R., Pirazzoli, L., Blasi, A., Bulgarelli, C., Hakuno, Y., Minagawa, Y., \& Brigadoi, S. (2019). Recommendations for motion correction of infant fNIRS data applicable to multiple data sets and acquisition systems. NeuroImage, 200, 511-527.

Dilks, D.D., Julian, J.B., Paunov, A.M., \& Kanwisher, N. (2013). The occipi- tal place area is causally and selectively involved in scene perception. Journal of Neuroscience, 33, 1331-1336.

Emberson, L. L., Cannon, G., Palmeri, H., Richards, J. E., \& Aslin, R. N. (2017). Using fNIRS to examine occipital and temporal responses to stimulus repetition in young infants: Evidence of selective frontal cortex involvement. Developmental Cognitive Neuroscience, 23, 26-38.

Esterman, M., Tamber-Rosenau, B. J., Chiu, Y. C., \& Yantis, S. (2010). Avoiding nonindependence in fMRI data analysis: leave one subject out. NeuroImage, 50(2), 572576. 
Fox, N. A., Rubin, K. H., Calkins, S. D., Marshall, T. R., Coplan, R. J., Porges, S. W., ... \& Stewart, S. (1995). Frontal activation asymmetry and social competence at four years of age. Child Development, 66(6), 1770-1784.

Gelman, A., \& Loken, E. (2013). The garden of forking paths: Why multiple comparisons can be a problem, even when there is no "fishing expedition" or "p-hacking" and the research hypothesis was posited ahead of time. Department of Statistics, Columbia University.

Gervain, J., Mehler, J., Werker, J.F., Nelson, C.A., Csibra, G., Lloyd-Fox, S., ... Aslin, R.N. (2011). Near-infrared spectroscopy: A report from the McDonnell infant methodology consortium. Developmental Cognitive Neuroscience, 1, 22-46.

Goodwin, J. R., Cannaday, A. E., Palmeri, H. G., Di Costanzo, A., Emberson, L. L., Aslin, R. N., \& Berger, A. J. (2016). Methodology for high-yield acquisition of functional nearinfrared spectroscopy data from alert, upright infants. Neurophotonics, 3(3), 031415.

Grossmann, T., Johnson, M. H., Lloyd-Fox, S., Blasi, A., Deligianni, F., Elwell, C., \& Csibra, G. (2008). Early cortical specialization for face-to-face communication in human infants. Proceedings of the Royal Society B: Biological Sciences, 275(1653), 28032811.

Hu, X. S., Arredondo, M. M., Gomba, M., Confer, N., DaSilva, A. F., Johnson, T. D., ... \& Kovelman, I. (2015). Comparison of motion correction techniques applied to functional near-infrared spectroscopy data from children. Journal of Biomedical Optics, 20(12), 126003.

Huppert, T.J., Diamond, S.G., Franceschini, M.A., \& Boas, D.A. (2009). HomER: A review of time-series analysis methods for near-infrared spectroscopy of the brain. Applied Optics, 48, D280-D298.

Hyde, D. C., Boas, D. A., Blair, C., \& Carey, S. (2010). Near-infrared spectroscopy shows right parietal specialization for number in pre-verbal infants. NeuroImage, 53(2), 647 652.

Issard, C., \& Gervain, J. (2018). Variability of the hemodynamic response in infants: Influence of experimental design and stimulus complexity. Developmental Cognitive Neuroscience, 33, 182-193. 
Jahani, S., Setarehdan, S. K., Boas, D. A., \& Yücel, M. A. (2018). Motion artifact detection and correction in functional near-infrared spectroscopy: a new hybrid method based on spline interpolation method and Savitzky-Golay filtering. Neurophotonics, 5(1), 015003.

Krueger, F., McCabe, K., Moll, J., Kriegeskorte, N., Zahn, R., Strenziok, M., ... \& Grafman, J. (2007). Neural correlates of trust. Proceedings of the National Academy of Sciences, 104(50), 20084-20089.

Lloyd-Fox, S., Blasi, A., Volein, A., Everdell, N., Elwell, C.E., \& Johnson, M.H. (2009). Social perception in infancy: A near infrared spectroscopy study. Child Development, 80, 986-999.

Malonek, D., \& Grinvald, A. (1996). Interactions between electrical activity and cortical microcirculation revealed by imaging spectroscopy: implications for functional brain mapping. Science, 272, 551-554.

Meek, J.H., Firbank, M., Elwell, C.E., Atkinson, J., Braddick, O., \& Wyatt, J.S. (1998). Regional hemodynamic responses to visual stimulation in awake infants. Pediatric Research, $43,840-843$.

Molavi, B., \& Dumont, G. A. (2012). Wavelet-based motion artifact removal for functional near-infrared spectroscopy. Physiological Measurement, 33(2), 259-270.

Naoi, N., Minagawa-Kawai, Y., Kobayashi, A., Takeuchi, K., Nakamura, K., Yamamoto, J. I., \& Shozo, K. (2012). Cerebral responses to infant-directed speech and the effect of talker familiarity. NeuroImage, 59(2), 1735-1744.

Otsuka, Y., Nakato, E., Kanazawa, S., Yamaguchi, M.K., Watanabe, S., \& Kakigi, R. (2007). Neural activation to upright and inverted faces in infants measured by near infrared spectroscopy. NeuroImage, 34, 399-406.

Pitcher, D., Dilks, D.D., Saxe, R.R., Triantafyllou, C., \& Kanwisher, N. (2011). Differential selectivity for dynamic versus static information in face- selective cortical regions. NeuroImage, 56, 2356-2363.

Powell, L.J., Deen, B. \& Saxe, R. (2018). Using individual functional channels of interest to study cortical development with fNIRS. Developmental Science, 21, e12595.

Powell, L.J., Kosakowski, H.L., Spelke, E.S. \& Saxe, R. (2017). Investigating the nature of infants' preference for imitators using neural and behavioral measures. Poster 
presented at the Meeting of the Cognitive Development Society, Portland, OR.

Powell, L.J. \& Spelke, E.S. (2017, September 30). The role of perceptual access in infants' third party evaluation of imitation. https://psyarxiv.com/63tyq/

Powell, L.J. \& Spelke, E.S. (2018). Third party preferences for imitators in preverbal infants. Open Mind, 2, 61-71.

Reyes, L. M. D., Bohache, K., Wijeakumar, S., \& Spencer, J. P. (2018). Evaluating motion processing algorithms for use with functional near-infrared spectroscopy data from young children. Neurophotonics, 5(2), 025008.

Scholkmann, F., Spichtig, S., Muehlemann, T., \& Wolf, M. (2010). How to detect and reduce movement artifacts in near-infrared imaging using moving standard deviation and spline interpolation. Physiological Measurement, 31(5), 649-662.

Sheth, S. A., Nemoto, M., Guiou, M., Walker, M., Pouratian, N., \& Toga, A. W. (2004). Linear and nonlinear relationships between neuronal activity, oxygen metabolism, and hemodynamic responses. Neuron, 42(2), 347-355.

Simmons, J. P., Nelson, L. D., \& Simonsohn, U. (2011). False-positive psychology: Undisclosed flexibility in data collection and analysis allows presenting anything as significant. Psychological Science, 22(11), 1359-1366.

Turk-Browne, N. B., Scholl, B. J., Chun, M. M., \& Johnson, M. K. (2009). Neural evidence of statistical learning: Efficient detection of visual regularities without awareness. Journal of Cognitive Neuroscience, 21(10), 1934-1945.

Van Den Bos, W., McClure, S. M., Harris, L. T., Fiske, S. T., \& Cohen, J. D. (2007). Dissociating affective evaluation and social cognitive processes in the ventral medial prefrontal cortex. Cognitive, Affective, \& Behavioral Neuroscience, 7(4), 337-346.

Werchan, D. M., Collins, A. G., Frank, M. J., \& Amso, D. (2016). Role of prefrontal cortex in learning and generalizing hierarchical rules in 8-month-old infants. Journal of Neuroscience, 36(40), 10314-10322.

Wilcox, T., Bortfeld, H., Woods, R., Wruck, E., \& Boas, D.A. (2005). Using near-infrared spectroscopy to assess neural activation during object processing in infants. Journal of Biomedical Optics, 10, 011010. 
Yücel, M. A., Selb, J., Cooper, R. J., \& Boas, D. A. (2014). Targeted principle component analysis: a new motion artifact correction approach for near-infrared spectroscopy. Journal of Innovative Optical Health Sciences, 7(02), 1350066. 